\title{
Theory of Auger energies in free atoms: Application to the alkaline earths
}

\author{
Donald R. Beck \\ Physics Department, Michigan Technological University, Houghton, Michigan 49931 \\ C. A. Nicolaides \\ Theoretical and Physical Chemistry Institutes, National Hellenic Research Foundation, \\ 48 Vasileos Constantinou, Athens 11635, Greece
}

(Received 9 December 1985)

\begin{abstract}
We calculate, including correlation, relativistic, and radiative effects, the Auger energies associated with the $K L_{2,3} L_{2,3}\left({ }^{3} P,{ }^{1} D_{2},{ }^{1} S_{0}\right)$ transitions in $\mathrm{Mg}$ to be, respectively, $1171.25,1166.9,1161.9 \mathrm{eV}$. The experimental values are, respectively, 1171.0 (1170.85), $1167.1(1167.0)$, and $1161.9(1161.75) \mathrm{eV}$. For the $K L_{2,3} L_{2,3}\left({ }^{1} D_{2}\right)$ transition in $\mathrm{Ca}$, we obtain $3275.8 \mathrm{eV}$ and for the $\mathrm{Be} K L_{1} L_{1}\left({ }^{1} S_{0}\right)$ transition, $96.21 \mathrm{eV}$, in good agreement with the experimental value of $96.11 \mathrm{eV}$. Predictions are also made for $\mathrm{O}$ and $\mathrm{F} K L L$ Auger energies.
\end{abstract}

\section{INTRODUCTION}

Recent papers in Physical Review A have dealt with the theory and experiment of inner electron binding energies (BE's) and Auger energies (AE's) of free atoms. ${ }^{1-4}$ New experimental values were presented and comparisons were made with theoretical results.

The main theme of these papers was the importance of relativistic and/or correlation effects-something which has been pointed out in the earlier literature as well.

Aksela et al. ${ }^{1}$ used experimental values found in the literature or obtained in their laboratory. Extensive comparison was made with multiconfigurational Dirac-Fock calculations, obtained using the computer program of Grant et al. 5,6 They observed "considerable deviations" (from 1 to $7 \mathrm{eV}$ ), which they attributed to "the presence of electron correlation either in the initial or in the final state of the Auger decay." Some of the cases presented by Aksela et al. ${ }^{1}$ have been studied by us earlier ${ }^{7,8}$ and recently. ${ }^{9}$ A collection of results of binding energies of $K$ and $L$-shell electrons is presented in Table $I$.

Banna and Slaughter ${ }^{2}$ analyzed the $2 p$-core spectrum of atomic potassium and compared the binding energy of the $2 p_{1 / 2}$ and $2 p_{3 / 2}$ levels with previous experiments and the theoretical results of Beck and Nicolaides. ${ }^{7,8}$ These numbers are given in Table $\mathrm{I}$.

Chen et $a l .^{3}$ presented a series of results based on calculations at the relativistic Dirac-Fock-Slater level with corrections for magnetic-retardation, radiative, and electron correlation effects. The utility of such analyses has been demonstrated by Beck and Nicolaides ${ }^{7,10}$ and Nicolaides et al. ${ }^{11}$

Finally, Bruch et al. ${ }^{4}$ presented experimental and theoretical results on the $K$-shell BE's of B and C. Their approach was based on the use of the semiempirical pair energies of Oksuz and Sinanoglu. ${ }^{12}$ The theory and possibility of treating Auger states of open-shell systems in such a way was proposed and implemented by us in the early 1970 s. $^{13-15}$ The $1 s$ BE of carbon, which was predicted in Refs. 7 and 15, is included again in Table $I$.

Given the continuing interest in the accurate prediction of BE's and AE's, as exemplified by the recent Refs. 1-4, we have applied our many-electron theory of such quantities $^{7-11,13-15}$ to the calculation of Auger energies in the alkaline-earth metals $\mathrm{Be}, \mathrm{Mg}$, and $\mathrm{Ca}$. The predictions agree well with the experimental values for $\mathrm{Be}$ and $\mathrm{Mg}$. For $\mathrm{Ca}$, there are no experimental results yet.

\section{REVIEW OF THE THEORY OF INNER HOLE STATE AND OF AUGER ENERGIES}

Until the early 1970s, the theory of atomic Auger energies was developed and applied in terms of some model based on the independent-particle approximation with or without inclusion of relativistic effects (e.g., see Refs. 20-22 and references therein). The first integrated proposals for the systematic incorporation of many-electron effects, in addition to relativistic and radiative corrections, appeared in Refs. 13-15, 23, 24, 7, and 25.

Fundamental aspects of the Auger phenomenon and related calculations were reviewed recently by Aberg and Howat. $^{26}$ In this section, we will bring attention to only certain characteristics of the problems related to the electronic structure of autoionizing states and the corresponding calculation of the Auger energies. ${ }^{7-10,13-15}$ For more rigorous discussions and recent developments on the calculation of widths, the reader is referred to Refs. 11, 13, 19 , and 26-29.

\section{A. Theory of the nonrelativistic electronic structure of inner hole states}

The theory is state specific, i.e., it describes each state of interest with its own optimized zeroth-order wave function and its own, separately optimized, virtual function space. For a recent review of this approach with applications to the electronic structure and properties of excited atoms and molecules, see Ref. 30.

For an autoionizing state, the wave function is separated formally into two components:

$$
\psi(E)=a(E) \psi_{0}+\int b^{E}\left(E^{\prime}\right) X_{\mathrm{as}}^{E}\left(E^{\prime}\right) d E^{\prime}
$$


TABLE I. Some existing binding ( $K$ shell unless otherwise indicated in "Atom" column) and $K L L$ Auger energies for light atoms (in $\mathrm{eV}$ ).

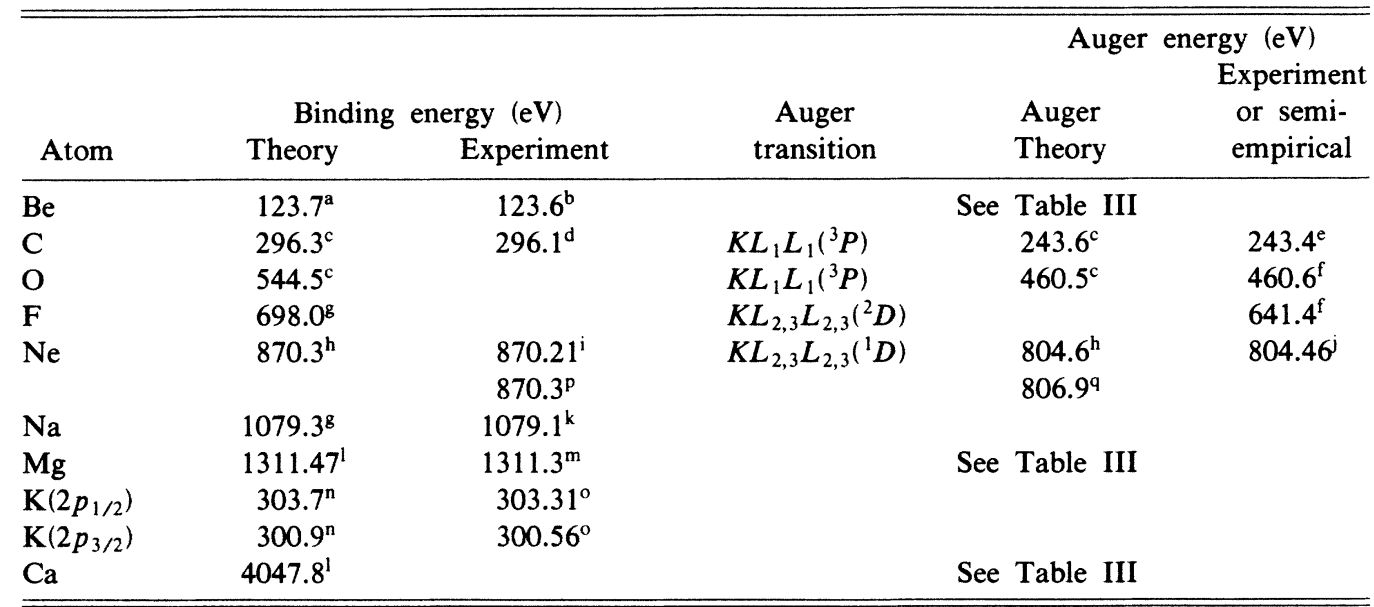

${ }^{a}$ Reference 11 .

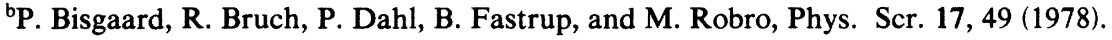

${ }^{\mathrm{c}}$ Reference 15.

${ }^{\mathrm{d}}$ Reference 4.

${ }^{e}$ Experimental, this work, using experimental [C. E. Moore, Atomic Energy Levels (U.S. GPO, Washington, D.C., 1949), Vol. 1] optical energy.

fSemiempirical; this work. Like footnote e, except theoretical binding energy was used.

${ }^{8}$ Reference 24.

${ }^{\mathrm{h}}$ References 23 and 19.

${ }^{\mathrm{i}}$ Reference 17.

${ }^{\mathrm{j}}$ Experimental, Ref. 1.

${ }^{k}$ M. S. Banna, B. Wallbank, D. C. Frost, C. A. McDowell, and J. S. H. Q. Perera, J. Chem. Phys. 68, 5459 (1978).

'Reference 9.

${ }^{\mathrm{m}}$ H. Agren, J. Nordgren, L. Selander, C. Nordling, and K. Siegbahn, J. Electron Spectros. Relat. Phenom. 14, 27 (1978); W. Mehlhorn, B. Breuckmann, and D. Hausamann, Phys. Scr. 16, 177 (1979).

See also Ref. 16.

"Reference 8.

${ }^{\circ}$ Reference 2.

${ }^{\mathrm{P}}$ Reference 18.

${ }^{\mathrm{q}}$ Ch. Briancon and J. P. Desclaux, Phys. Rev. A 13, 2157 (1976).

$\psi_{0}$ is bound and $X_{\mathrm{as}}^{E}\left(E^{\prime}\right)$ contains the asymptotic, energynormalized terms which represent the open channels. Although this separation is naturally dictated by scattering theory and forms the basis for the fundamental analysis of spectroscopic phenomena, ${ }^{26,31}$ the relevant question here is how to obtain $\psi_{0}$ and $X_{\text {as }}(E)$, and how to analyze them into important or unimportant terms for each observable.

The overwhelming contribution to the total energy comes from $\psi_{0}$. Nevertheless, the contribution of the second component to the Auger energy should, in principle, also be calculated, especially since an Auger transition often occurs between two autoionizing states (i.e., the final state could itself be broadened by autoionization). However, for the $K L L$ Auger transitions studied here, its effect on the transition energy is very small and will not be considered (for example, Nicolaides et al. found ${ }^{19}$ that for the $\mathrm{Ne}^{+} 1 s 2 s^{2} 2 p^{6}$ Auger state, the energy shift, $\Delta$, due to open-channel mixing, is $-0.1 \mathrm{eV}$, while its total energy is $870.3 \mathrm{eV}$ above the ground state and the total width is about $0.25 \mathrm{eV}$ ).

$\psi_{0}$ is the square-integrable component describing the initially localized state. Its consistent evaluation for an arbitrary system requires the correct determination of a zeroth-order bound wave function which contains the strongly mixed bound configurations obtained selfconsistently. This wave function is composed of the Fermi-sea orbitals which are specific to each state of interest. $^{7,24,30}$ It is designated as $\Phi_{\mathrm{FS}}$ and is obtained numerically using the program of Froese Fischer ${ }^{32}$ under the constraints of the correct boundary conditions, number of nodes, satisfaction of the virial theorem, and orthogonality to zeroth-order Hartree-Fock orbitals of lower configurations corresponding to the open channels. ${ }^{13}$

The remaining localized components of $\psi_{0}$-which contribute to the stability of the state-are obtained variationally by optimizing the single-, pair-, triple-, etc. symmetry-adapted correlation functions which contain analytic Slater-type virtual orbitals. ${ }^{7}$ These correlation functions are constrained to be orthogonal to the same Hartree-Fock orbitals which make up the core of the open channels. ${ }^{13}$

Thus, the form of $\psi_{0}$ is

$$
\psi_{0}=\Phi_{\mathrm{FS}}+X_{\mathrm{loc}} \text {, }
$$


where $X_{10 c}$ represents all the localized correlation vectors. In practice, these contain one- and two-symmetry-adapted orbital excitations from $\Phi_{\mathrm{Fs}}{ }^{7}$ Higher-order excitations have only a small contribution to energy differences of the Auger type. ${ }^{33}$

The development of the theory of Auger energies along these lines has also contributed to the systematization of useful information as regards approximate cancellation or correlation beyond the state-specific $\triangle \mathrm{SCF}$ procedure $^{7,8,11,15}$ and the introduction of simple electron correlation rules which allow the prior recognition of the important correlation effects in excited states and in various spectroscopies across the Periodic Table. ${ }^{7-10,24,28,34,35}$ Apart from their calculational value, these rules can be used as a tool for the understanding of atomic spectra which do not conform to the independent-particle-model predictions.

We close this section by pointing out two more positive aspects of the state-specific approach. One refers to the use of the well-known $\triangle \mathrm{SCF}$ approximation the other to the prediction of accurate Auger energies using optical data for the final state.

(a) The state-specific approach also brings out the occasional advantages of the (uncorrelated) well-known term-dependent $\triangle \mathrm{SCF}$ procedure (which, of course, is state specific). For example, in the case of the $1 s$ binding energy of $\mathrm{Be}$ (Ref. 11), where the main effect is relaxation, the $\triangle \mathrm{SCF}$ result $(123.34 \mathrm{eV})$ is in much better agreement with experiment $(123.6 \pm 0.1 \mathrm{eV})$ than are two manyelectron methods [Green's function (124.5 eV, Ref. 36) and extended Koopmans's (127.9 eV, Ref. 37)] which study the $K$-shell excitation from a correlated ground state.

Calculations at the $\triangle \mathrm{HF}$-SCF level (without any approximation for the exchange interaction) can be of much value for the prediction of core binding energies in metals, either through the "excitonic" model, ${ }^{7,38,39}$ or through $a b$ initio cluster calculations; ${ }^{40} \triangle \mathrm{SCF}$ calculations have also been used to evaluate a number of approximations used for the study of chemical shifts. ${ }^{15}$

(b) In order to avoid excessive computation for Auger energies, the use of optical data for the final state was introduced $^{15}$ in conjunction with ab initio state-specific calculations of the initial state. Comparison with experiments using the same optical data, would then test the initial-state binding energy. For C $K L L$, our early value ${ }^{15}$ was $243.6 \mathrm{eV}$, while utilization of a recent experiment's ${ }^{4}$ binding energy yields $243.4 \mathrm{eV}$. For the $\mathrm{Ne} K L L\left({ }^{1} \mathrm{D}\right)$ Auger energy, we predicted ${ }^{24} 804.6 \mathrm{eV}$, in agreement with the experimental value ${ }^{18}$ of $804.557 \pm 0.017 \mathrm{eV}$. Aksela et al. ${ }^{1}$ quote a theoretical result for this Auger energy of $806.78 \mathrm{eV}$. As Aberg and Howat have also suggested, ${ }^{26}$ the combination of theoretical predictions for the initial state with optical data for the final state can prove a useful and economic means for studying inner hole excited states. Table I also contains predictions for $\mathrm{O}$ and $\mathrm{F} K L L$ Auger energies using this method.

\section{B. Relativistic theory and radiative corrections}

Given the absence of a completely relativistic, manyelectron theory in Hamiltonian form and the limitations of applying quantum electrodynamics accurately beyond one-electron atoms, ${ }^{41}$ these effects must be incorporated in the calculation of binding or Auger energies via some approximation.

Our proposal and implementation for the incorporation of radiative effects is presented in Refs. 7 and 23. It is based on the appropriate scaling of the QED results on one-electron ions. ${ }^{42,43}$

Relativistic effects are computed at the multiconfigurational Dirac-Fock (MCDF) level using the computer program of Desclaux. ${ }^{44}$ Regarding the analysis given above, this involves the formal replacement of $\Phi_{\mathrm{FS}}$ of $\mathrm{Eq}$. (2) with $\Phi_{\mathrm{RFS}}$. The rigorous incorporation of relativistic correlation using at least the Breit operator is an open subject. An outline of the extension of our theory, now in progress, has been given in Ref. 7(b). Special emphasis on the appropriate choice of electron function spaces was given. As was pointed out in Ref. 35(b), in analogy with the autoionization problem and the orthogonality and boundary conditions imposed there on the one- and many-electron bound functions, ${ }^{13}$ the use of Dirac-Fock one-positron projection operators on the relativistic correlation functions is expected to alleviate problems of "variational collapse" into the positron continuum ${ }^{45}$ with sufficient accuracy. Orthogonality to Dirac-Fock positron orbitals rather than to some higher-order, correlated positron functions is rigorously justified to second order in perturbation theory-in analogy with what has been proven for autoionizing many-electron states. ${ }^{13}$ Analysis and discussions on the need of defining projected relativistic operators have been given by Mittleman ${ }^{46}$ and by Sucher. $^{47}$ For example, Mittleman ${ }^{46}$ has derived the DiracFock projection operators in relation to practical configuration-space Hamiltonians, which were then used to justify current relativistic calculations of the DiracFock type.

\section{METHOD OF APPLICATION AND RESULTS}

As is usual in our treatment of binding and Auger energies, the localized nonrelativistic correlation effects are broken into several units which are treated separately. This serves three purposes: (1) Some units are essentially identical in the initial and final states, and so are not calculated at all. For example, by using the ground state of the neutral atom as reference, the $1 s^{2}$ pair energy may be taken to be the same for it and the Auger final state. (2) A unit is chosen so as to minimize the number of virtual radial functions required (here, there are two virtual radials per $l$ ). This, for example, is characteristic of all excitations arising from the same shells. (3) Fragmentation into units tends to reduce the number of pair-pair interactions included, which would otherwise have to be balanced by including higher-order excitations.

In Table II, we present the various contributions to the energy difference between the ground state of $\mathrm{Mg}$ and the three final states associated with the Auger process, $\mathbf{M g}^{2+}$ $1 s^{2} 2 s^{2} 2 p^{4} 3 s^{2}\left({ }^{3} P,{ }_{1}^{1} D,{ }^{1} S\right)$. The first row contains the nonrelativistic single-configuration numerical Hartree-Fock (RHF) results obtained from the program of Froese Fisher. ${ }^{32}$ Following this, a relativistic calculation is performed 
TABLE II. Contributions (in a.u.) to the $K L_{2,3} L_{2,3}$ Auger energy of $\mathrm{Mg}$.

\begin{tabular}{lcccc}
\hline \hline Quantity & & & $E\left(\mathrm{Mg}^{2+}\right)$ & \\
${ }^{1} D_{2}$ & ${ }^{1} S_{0}$ \\
\hline RHF & $E(\mathrm{Mg})^{\mathrm{b}}$ & ${ }^{3} P_{2}$ & -194.394053 & -194.144938 \\
Relativistic & -199.614637 & -194.561527 & -0.293719 & -0.293390 \\
$\Delta$ QED & -0.288666 & -0.297488 & +0.007 & +0.007 \\
Internal & +0.007 & +0.007 & -0.042675 & -0.042392 \\
$\varepsilon\left(3 s^{2}\right)$ & -0.021652 & -0.042600 & -0.002672 & -0.002989 \\
$\varepsilon(L, L)$ & -0.012014 & -0.002790 & -0.133041 & -0.157342 \\
$2 s^{2} \rightarrow 2 p^{2}$ & -0.263426 & -0.124152 & & -0.084384 \\
$\varepsilon(L, 3 s)$ & & & -0.0305464 & -0.031714 \\
$\varepsilon(1 s, L)$ & -0.018173 & -0.032367 & -0.025437 & -0.025133 \\
Polarization & -0.029328 & -0.025114 & -0.055729 & -0.00722 \\
Total & 0.0 & -0.054198 & -194.965877 & -194.782502 \\
\hline \hline
\end{tabular}

asee text for explanations.

brom Ref. 9.

with the program of Desclaux, ${ }^{44}$ using a term-dependent electrostatic energy and a finite nucleus model. Two-body relativistic effects (Breit interaction) are evaluated to first order, using the average energy expression. As might be expected, the differential effects among the terms are much smaller than those between $\mathrm{Mg}$ and $\mathrm{Mg}^{2+}$

The radiative effects, $\Delta Q E D$, are referenced to $\mathrm{Mg}^{+}$as in Ref. 9, and account for the effect of the Lamb shift and vacuum polarization on a single screened $1 s$ electron. Using the work of Huang et al., ${ }^{48}$ we estimate the differential $2 p$ radiative effects to be below $20 \mu \mathrm{H}$, and so ignore them.

The first of the nonrelativistic correlation effects, $3 s^{2} \rightarrow 3 p^{2}$ (labeled "internal" in the table), is seen to be highly dependent on the ionization stage, as was noted elsewhere. ${ }^{9,11}$ For full comparison, the Auger initial state $\mathrm{had}^{9}$ an internal contribution of -0.032651 a.u. The remaining part of the all-valence-shell $\varepsilon\left(3 s^{2}\right)$ correlation shows a strong ionization dependence (for $\mathrm{Mg}^{+}$it is ${ }^{9}$ -0.008947 a.u.), but is small enough so the differential effects are modest.

The next entry, $\varepsilon(L, L)$, arises from double excitations out of the $L$ shell into either an occupied and virtual radial $\left(2 s^{2} \rightarrow 2 p V_{p}\right.$, an example ${ }^{7}$ of hole-virtual correlation) or two virtuals. The latter (bivirtual) falls into three categories, classified according to their origin, viz., $2 s^{2} \rightarrow$, $2 s 2 p \rightarrow$, and $2 p^{2} \rightarrow$. These bivirtual energies can be expressed $^{49}$ as a sum of products of "group factors" and radial pair energies which vary slowly with term, and somewhat less so with stage of ionization, etc. For $2 s^{2}$, the group factors are the same ${ }^{49}$ for $\mathbf{M g}, \mathrm{Mg}^{2+}$ (all terms), and for $\mathrm{Mg}^{+}\left(\mathrm{K}\right.$-hole). For $2 s 2 p, \mathrm{Mg}$ and $\mathrm{Mg}^{+}$have the same group factors, whereas those for all $\mathrm{Mg}^{2+}$ terms are $\frac{2}{3}$ of the former. ${ }^{49}$ To this point, then, $\varepsilon(L, L)$ only varies for the different terms of $\mathbf{M g}^{2+}$ due to the variation of "radial pair energies."

For the remaining part, in $\mathrm{Mg}^{+}$and $\mathrm{Mg}$, the group factors associated with the radial pair energies $\varepsilon\left(2 p^{23} P\right)$, $\varepsilon\left(2 p^{21} D\right)$, and $\varepsilon\left(2 p^{21} S\right)$ are, ${ }^{49}$ respectively, 9,5 , and 1 . For $\mathbf{M g}^{2+}$, however, these factors are term dependent. For ${ }^{3} P$ they are, respectively, $4, \frac{5}{3}$, and $\frac{1}{3}$; for ${ }^{1} D, 3, \frac{8}{3}$, and $\frac{1}{3}$ and for ${ }^{1} S, 3, \frac{5}{3}$, and $\frac{4}{3}$. The value of this is knowing, via calculated results, that $\bar{\varepsilon}\left(2 p^{23} P\right)=-0.243 \mathrm{eV}$, $\bar{\varepsilon}\left(2 p^{21} D\right)=-0.397 \mathrm{eV}$ and $\bar{\varepsilon}\left(2 p^{2}{ }^{1} S\right)=-1.17 \mathrm{eV}$, quantities which are, to a large extent, independent of term. To summarize, the variation among $\varepsilon(L, L)$ for the various terms is fundamentally due to the change in the group factors associated with $2 p^{2} \rightarrow$. The radial pair energies are not sufficiently invariant to degree of ionization to ascribe the principal variation in $\varepsilon(L, L)$ there to the group factors, however. Both the variation in $\varepsilon(L, L)$, and the internal correlation $2 s^{2} \rightarrow 2 p^{2}$ which exists only for ${ }^{1} S$ of $\mathrm{Mg}^{2+}$ (shown in the next row) are differentially quite important contributors to the Auger energy.

For $\varepsilon(L, 3 s)$ and $\varepsilon(1 s, L)$ there is no difference ${ }^{49}$ in the group factors for the various terms of $\mathrm{Mg}^{2+}$. Hence, any change is due to the radial factors, and, as can be seen from the table, this is small. On the other hand, for $\mathbf{M g}$, these contributions should be $\frac{3}{2}$ times larger on the basis of the group factors. As this is not so, we can see intershell radial factors can depend strongly on ionization as has been observed elsewhere. ${ }^{9}$

The remaining contributions arise from the polarizations (single excitation to virtual subshells). By far, the largest contributions arise from $2 s \rightarrow V_{d}$ and, to a lesser extent, from $2 p \rightarrow V_{f}$, as can be observed from the table, where these are absent in the ${ }^{1} S$ term.

The remaining excitations are those which violate Brillouin's theorem. For $\mathrm{Mg}^{+}$, these were found to be much larger ${ }^{9}(\sim-0.022$ a.u. total). What accounts for this difference? For both $\mathrm{Mg}^{+}$and $\mathrm{Mg}^{2+}{ }^{3} \mathrm{P}$, the $2 s \rightarrow V_{s}$ is the largest. In the former, the off-diagonal matrix element is $-\frac{3}{2} R^{0}\left(1 s 2 s ; V_{s} 1 s\right)=-0.3429$ a.u. and in the latter, $\frac{2}{3} R^{1}\left(2 s 2 p ; 2 p V_{s}\right)=0.1038$ a.u., and it is this factor which accounts for the relative decline of the effect in $\mathrm{Mg}^{2+}$.

Using the total energy for $\mathrm{Mg}^{+}$given in Ref. 9, we establish our results for the $K L_{2,3} L_{2,3}$ Auger energies which we present in Table III, together with the experimental values of Aksela et al. ${ }^{1}$ As can be seen from the table, our results are in excellent agreement with the available experimental values. ${ }^{1,50}$ Using the low- $Z$ Pauli approximation, ${ }^{51}$ and the RHF wave function, the fine-structure splitting for the ${ }^{3} P$ terms is ${ }^{3} P_{0^{-}}{ }^{3} P_{1}=0.0999 \mathrm{eV}$, and ${ }^{3} P_{1}$ ${ }^{3} P_{2}=0.215 \mathrm{eV}$. The ${ }^{3} P$ weighted average energy is then $0.153 \mathrm{eV}$ above the ${ }^{3} P_{2}$ level, so that the weighted Auger 
TABLE III. Auger energies in the alkaline-earth metals.

\begin{tabular}{llcl}
\hline \hline & & \multicolumn{2}{c}{ Auger energy $(\mathrm{eV})^{\mathrm{a}}$} \\
Species & \multicolumn{1}{c}{ Final state } & Theory & Experiment \\
\hline $\mathrm{Mg}^{2+}$ & $K L_{2,3} L_{2,3}\left({ }^{3} P_{2}\right)$ & $1171.4^{\mathrm{b}}$ & $1171.0^{\mathrm{c}}$ \\
& & & $1170.85^{\mathrm{d}}$ \\
$\mathrm{Mg}^{2+}$ & $K L_{2,3} L_{2,3}\left({ }^{1} D_{2}\right)$ & $1166.9^{\mathrm{b}}$ & $1167.0^{\mathrm{d}}$ \\
& & & $1167.1^{\mathrm{c}}$ \\
$\mathrm{Mg}^{2+}$ & $K L_{2,3} L_{2,3}\left({ }^{1} S_{0}\right)$ & $1161.9^{\mathrm{b}}$ & $1161.75^{\mathrm{d}}$ \\
& & & $1161.9^{\mathrm{c}}$ \\
$\mathrm{Ca}^{2+}$ & $K L_{2,3} L_{2,3}\left({ }^{1} D_{2}\right)$ & $3275.8^{\mathrm{b}}$ & \\
$\mathrm{Be}^{2+}$ & $K_{1} L_{1}\left({ }^{1} S_{0}\right)$ & $96.21^{\mathrm{e}}$ & $96.1 \pm 0.1^{\mathrm{f}}$ \\
\hline \hline
\end{tabular}

${ }^{\mathrm{a}} 1$ a.u. $=27.211608 \mathrm{eV}$.

'This work. The average " $3 P^{\prime}$ " Auger energy is $1171.25 \mathrm{eV}$ (see text for details).

${ }^{\mathrm{c}}$ Reference 51.

${ }^{\mathrm{d}}$ Reference 1.

'See footnote $f$ of Table I.

fSee footnote b of Table I.

energy is $1171.25 \mathrm{eV}$. It is this value that is compared to experiment. We might observe that nonrelativistically, the $1 s$ hole state in $\mathrm{Mg}^{+}$may undergo an Auger transition to only the ${ }^{1} D_{2}$ or ${ }^{1} S_{0}$ final state. Transitions to the ${ }^{3} P_{2}$ would only be allowed due to relativistic effects. A portion of this is due to the admixture of ${ }^{1} D_{2}$ into the ${ }^{3} P_{2}$ state (the remaining part would be due to using a relativistic Hamiltonian in the decay matrix element-see Ref. 52). There is a similar admixture of ${ }^{1} S_{0}$ into ${ }^{3} P_{0}$. Analysis of the relativistic Hartree-Fock result suggests an admixture of 0.03 for the ${ }^{1} D_{2}$ component. Thus one predicts the ${ }^{3} P_{2}$ intensity to be $\sim \frac{1}{1000}$ of the ${ }^{1} D_{2}$ from this cause which is in agreement with earlier theoretical work. ${ }^{53-55}$ Experimentally, the ratio is more like 1/33; clearly, a more thorough theoretical investigation would be desirable.

In Table IV, the various contributions to the energy difference between the ground state of $\mathrm{Ca}$ and the $\mathrm{Ca}^{2+}$ $1 s^{2} 2 s^{2} 2 p^{4} 3 s^{2} 3 p^{6} 4 s^{2}{ }^{1} D$ Auger final state are given. Most entries were obtained in the same manner as they were for $\mathrm{Mg} / \mathrm{Mg}^{2+}$.

For $\Delta \mathrm{QED}$, however, we did reexamine the role of radiative corrections to the $2 p$ levels by using the results of Huang et al., ${ }^{48}$ and accounting for the varying occupancies of $2 p_{1 / 2}$ and $2 p_{3 / 2}$ in the relativistic configurations used to construct ${ }^{44}$ the ${ }^{1} D$ final state. While individual terms were significant, the net effect (Ca versus $\mathrm{Ca}^{2+}$ ) was less than $5 \mu \mathrm{H}$, and so can be ignored.

The calculation of $\varepsilon\left(4 s^{2}\right)$, which includes $4 s$ polarizations in $\mathrm{Ca}^{++}$, presented a somewhat novel feature. Due to the higher ionicity in $\mathrm{Ca}^{++}$, the $3 d$ drops below the $4 s$ (4s remains below $3 d$ in $\mathrm{Ca}$ and $\mathrm{Ca}^{+}$for the configurations of interest), which resulted in four configurations, viz., $2 p^{4}\left({ }^{3} P\right) 4 s 3 d\left({ }^{3} D\right), 2 p^{4}\left({ }^{1} D\right) 3 d^{2}\left({ }^{1} S\right), 2 p^{4}\left({ }^{1} D\right) 3 d 4 s\left({ }^{1} D\right)$, and $2 p^{4}\left({ }^{1} D\right) 3 d 4 d\left({ }^{1} S\right)$, being below $2 p^{4}\left({ }^{1} D\right) 4 s^{2}\left({ }^{1} S\right)$. There are actually quite a few others, but these do not directly connect to our RHF vector, and are thus ignored.

So we have the situation of a perturber (namely the RHF) embedded in several Rydberg series. As has been noted in the past, ${ }^{56}$ it is difficult to find a common orthonormal set of radial functions that properly describes both
TABLE IV. Contributions (in a.u.) to the $K L_{2,3} L_{2,3}\left({ }^{1} D_{2}\right)$ Auger energy of $\mathrm{Ca}$.

\begin{tabular}{lcc}
\hline \hline Quantity $^{\mathrm{a}}$ & $E(\mathrm{Ca})^{\mathrm{b}}$ & $E\left(\mathrm{Ca}^{2+}{ }^{1} D_{2}\right)^{\mathrm{c}}$ \\
\hline RHF & -676.758186 & -648.407936 \\
Relativistic & -2.761420 & -2.748203 \\
$\Delta$ QED & +0.05068 & +0.05068 \\
Internal & -0.019415 & -0.0345719 \\
$\varepsilon\left(4 s^{2}\right)$ & -0.009052 & -0.001552 \\
$\varepsilon(L, L)$ & $-0.246463^{\mathrm{c}}$ & -0.113782 \\
$\varepsilon(L, M)$ & $-0.0751863^{\mathrm{c}}$ & -0.0742042 \\
$\varepsilon(K, L)$ & -0.029451 & -0.030345 \\
$\varepsilon(M, 4 s)$ & -0.026466 & -0.0585066 \\
Polarization & 0.0 & -0.087789 \\
Total & -679.874959 & -651.50621 \\
\hline \hline
\end{tabular}

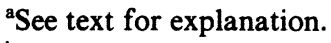

${ }^{b}$ From Ref. 9, unless otherwise indicated.

'This work.

off-diagonal and diagonal matrix elements. Here we have chosen to adopt an ad hoc procedure like the one used earlier ${ }^{56}$-we use all the radials of the RHF function, a $3 d$ from " $3 d^{2}$," and a $4 d$ from " $3 d 4 d$," which is then orthogonalized to the $3 d$. Separate RHF runs on the lower four configurations are performed to establish the amount of energy to be added to each of the diagonal matrix elements computed from the common orthonormal set of configurations below the RHF solution (the socalled $^{56}$ Down Shift Model or DSM). A few additional Rydberg functions, representing nearby higher configurations, were then added and shifted, and finally virtuals, which were iterated.

One may inquire whether there is a significant probability that a more energetic Auger electron would be emitted, with a concomitant energetically lower final state, e.g., how probable is $2 p^{4}\left({ }^{1} D\right) 3 d^{2}\left({ }^{1} S\right)$ ? Since none of the squares of the coefficients of the four lower configurations exceeds 0.01 , we regard it as fairly unlikely.

The large relative size of $\varepsilon(M, 4 s)$ correlation is also in part due to the greater importance of the $3 d$, but here the RHF result is the lowest state of interest, so no special techniques need be applied. Finally, as remarked earlier, ${ }^{9}$ future work might include examining the extent of nontransferability of the purely $\boldsymbol{M}$-shell correlation.

Our prediction for the $K L_{2,3} L_{2,3}\left({ }^{1} D_{2}\right)$ Auger energy for $\mathrm{Ca}$ is obtained using the theoretical binding energy of Ref. 9, and is given in Table III. The $K L_{1} L_{1}\left({ }^{1} S_{0}\right)$ Auger energy of $\mathrm{Be}$ is also shown in the table, and was obtained using the theoretical binding energy ${ }^{11}$ and optical measurement.

It can be seen that where experimental values exist, theory and experiment are in excellent $(\leq 0.2-\mathrm{eV}$ difference) agreement, and that even theoretical values of ten years ago have stood the test of time rather well.

\section{ACKNOWLEDGMENTS}

One of us (D.R.B.) wishes to thank the National Science Foundation for partial support of this work (under Grants No. PRM-81-11589 and No. PHY-85-06177). 
${ }^{1}$ H. Aksela, S. Aksela, and H. Patana, Phys. Rev. A 30, 858 (1984).

${ }^{2}$ M. S. Banna and A. R. Slaughter, Phys. Rev. A 30, 3021 (1984).

${ }^{3}$ M. H. Chen, B. Crasemann, N. Mårtensson, and B. Johannson, Phys. Rev. A 31, 556 (1985).

${ }^{4}$ R. Bruch, W. L. Luken, J. C. Culberson, and K. T. Chung, Phys. Rev. A 31, 503 (1985).

5I. P. Grant, B. J. McKenzie, P. H. Norrington, D. F. Mayers, and N. C. Pyper, Comput. Phys. Commun. 21, 207 (1980).

${ }^{6}$ B. J. McKenzie, I. P. Grant, and P. H. Norrington, Comput. Phys. Commun. 21, 233 (1980).

7(a) D. R. Beck and C. A. Nicolaides, in Excited States in Quantum Chemistry, edited by C. A. Nicolaides and D. R. Beck (Reidel, Dordrecht, 1978), p. 329; (b) see pp. 349-355 of this reference.

${ }^{8}$ D. R. Beck and C. A. Nicolaides, Phys. Rev. A 26, 857 (1982).

${ }^{9}$ D. R. Beck, J. Chem. Phys. 81, 5002 (1984).

10D. R. Beck and C. A. Nicolaides, Int. J. Quantum Chem. Symp. 14, 323 (1980).

${ }^{11}$ C. A. Nicolaides, Y. Komninos, and D. R. Beck, Phys. Rev. A 27, 3044 (1983).

12I. Oksuz and O. Sinanoglu, Phys. Rev. 181, 42 (1969); 181, 54 (1969).

${ }^{13}$ C. A. Nicolaides, Phys. Rev. A 6, 2078 (1972).

${ }^{14}$ C. A. Nicolaides, Chem. Phys. Lett. 19, 69 (1973).

${ }^{15}$ C. A. Nicolaides and D. R. Beck, Chem. Phys. Lett. 27, 269 (1974).

${ }^{16}$ M. S. Banna, A. R. Slaughter, R. D. Mathews, R. J. Key, and S. M. Ballina, Chem. Phys. Lett. 92, 122 (1982).

${ }^{17}$ L. Petterson, J. Nordgren, L. Selander, C. Nordling, and $\mathbf{K}$. Siegbahn, J. Electron Spectrosc. 27, 29 (1982).

${ }^{18}$ T. D. Thomas and R. W. Shaw, J. Electron Spectrosc. 8, 45 (1976).

${ }^{19}$ C. A. Nicolaides, Th. Mercouris, and Y. Komninos, Int. J. Quantum Chem. 26, 1017 (1984).

${ }^{20}$ E. H. S. Burhop and W. N. Asaad, Adv. At. Mol. Phys. 8, 163 (1972).

${ }^{21}$ W. N. Asaad and D. Petrini, Proc. R. Soc. London, Ser. A 350, 381 (1976).

22F. P. Larkins, in Atomic Inner-Shell Processes, edited by B. Crasemann (Academic, New York, 1975), p. 377.

${ }^{23}$ D. R. Beck and C. A. Nicolaides, J. Electron Spectros. Relat. Phenom. 8, 249 (1976).

${ }^{24}$ D. R. Beck and C. A. Nicolaides, Int. J. Quantum Chem. Symp. 8, 17 (1974); 10, 119 (1976).

${ }^{25}$ H. P. Kelly, Phys. Rev. A 11, 556 (1975).

${ }^{26}$ T. Aberg and G. Howat, in Handbuch der Physik, edited by W. Mehlhorn (Springer-Verlag, Berlin, 1982), Vol. 31.

${ }^{27}$ C. A. Nicolaides and D. R. Beck, Int. J. Quantum Chem. 14, 435 (1978).
${ }^{28}$ Y. Komninos, A. Aspromallis, and C. A. Nicolaides, Phys. Rev. A 27, 1865 (1983).

${ }^{29}$ G. Aspromallis, C. A. Nicolaides, and Y. Komninos, J. Phys. B 18, L545 (1985).

${ }^{30} \mathrm{C}$. A. Nicolaides, in Advanced Theories and Computational Approaches to the Electronic Structure of Molecules, edited by C. Dykstra (Reidel, Dordrecht, 1984), p. 161.

${ }^{31}$ U. Fano, Phys. Rev. 124, 1866 (1961).

${ }^{32}$ C. Froese Fischer, Comput. Phys. Commun. 4, 107 (1972).

${ }^{33} \mathrm{We}$ point out that for certain excited states, the existence of an inner hole causes higher-order correlations to be relatively more important (e.g., see Ref. 30 for the effect of triple excitations in $\mathrm{Cl} K L 3 s 3 p^{62} S$ ).

${ }^{34}$ C. A. Nicolaides and D. R. Beck, Chem. Phys. Lett. 36, 79 (1975).

35(a) C. A. Nicolaides and D. R. Beck, in Excited States in Quantum Chemistry, Ref. 7, p. 143; (b) see p. 178 of this reference.

360. Walter and J. Shirmer, J. Phys. B 14, 3805 (1981).

${ }^{37}$ R. C. Morrison, Chem. Phys. Lett. 62, 131 (1979).

${ }^{38}$ L. Ley, S. P. Kowalczyk, F. R. McFeely, R. A. Pollak, and D. A. Shirley, Phys. Rev. B 8, 2392 (1973).

${ }^{39}$ R. E. Watson, M. C. Perlman, and J. F. Herbst, Phys. Rev. B 13, 2358 (1976).

${ }^{40}$ C. A. Nicolaides, A. Zdetsis, and A. Andriotis, Solid State Commun. 50, 857 (1984).

${ }^{41}$ For recent progress, see P. J. Mohr, Phys. Rev. A 32, 1949 (1985).

${ }^{42}$ G. W. Erickson, Phys. Rev. Lett. 27, 879 (1971).

${ }^{43}$ P. J. Mohr, in Relativistic Effects in Atoms, Molecules and Solids, edited by G. L. Malli (Plenum, New York, 1983), p. 145.

44J. P. Desclaux, Comput. Phys. Commun. 9, 31 (1975).

${ }^{45}$ G. E. Brown and D. G. Ravenhall, Proc. R. Soc. London, Ser. A 208, 552 (1951).

${ }^{46}$ M. H. Mittleman, Phys. Rev. A 24, 1167 (1981); 5, 2395 (1972).

47J. Sucher in Relativistic Effects in Atoms, Molecules and Solids, Ref. 43, p. 1; also see Phys. Rev. A 22, 348 (1980).

${ }^{48}$ K. Huang, M. Aoyagi, M. H. Chen, B. Crasemann, and H. Mark, At. Data. Nucl. Data Tables 18, 243 (1976).

${ }^{49}$ D. R. Beck and C. A. Nicolaides, in Excited States in Quantum Chemistry, Ref. 7, p. 105.

${ }^{50}$ B. Breuckmann, J. Phys. B 12, L609 (1979).

${ }^{51}$ D. R. Beck, J. Chem. Phys. 51, 2171 (1969).

${ }^{52} \mathrm{G}$. Aspromallis, C. A. Nicolaides, and Y. Komninos, J. Phys. B 18, L545 (1985).

${ }^{53}$ E. McGuire, Phys. Rev. 185, 1 (1969).

${ }^{54}$ D. C. Walters and C. P. Bhalla, At. Data. 3, 301 (1971).

${ }^{55}$ M. H. Chen and B. Crasemann, Phys. Rev. A 8, 7 (1973).

56D. R. Beck and C. A. Nicolaides, Phys. Lett. 61A, 227 (1977). 